\title{
Distributed Saturated Control for a Class of Semilinear PDE Systems: A SOS Approach
}

\author{
José Luis Pitarch, Mohsen Rakhshan, Mohammad Mehdi Mardani and Mokhtar Shasadeghi
}

\begin{abstract}
This paper presents a systematic approach to deal with the saturated control of a class of distributed parameter systems which can be modeled by first-order hyperbolic partial differential equations (PDE). The approach extends (also improves over) the existing fuzzy Takagi-Sugeno (TS) state feedback designs for such systems by applying the concepts of the polynomial sum-of-squares (SOS) techniques. Firstly, a fuzzy-polynomial model via Taylor series is used to model the semilinear hyperbolic PDE system. Secondly, the closed-loop exponential stability of the fuzzy-PDE system is studied through the Lyapunov theory. This allows to derive a design methodology in which a more complex fuzzy state-feedback control is designed in terms of a set of SOS constraints, able to be numerically computed via semidefinite programming. Finally, the proposed approach is tested in simulation with the standard example of a nonisothermal plug-flow reactor (PFR).
\end{abstract}

Index Terms-Hyperbolic PDE, Fuzzy Polynomial, SOS, Input Saturation, Distributed-parameter Systems

\section{INTRODUCTION}

Numerous processes in industry are essentially distributed in space, i.e., their behavior is determined by three-dimensional position in addition to time [1]. Fundamental thermodynamics allows to obtain mathematical models for such processes: after applying the balance equations (mass, energy and momentum), the obtained models are usually in PDE form. Furthermore, industrial processes are inherently complex, leading to semilinear, quasilinear or full-nonlinear PDE systems [2].

In general, PDE systems can be classified in three classes according to the behavior of their characteristic equation: hyperbolic, parabolic and elliptic [3]. In particular, hyperbolic PDE systems represent the dynamics of some industrial processes which are used in convection with negligible diffusion effects, such as fluid heat exchangers, tubular reactors, and fiber spin lines. Because the PDE systems are inherently infinite-dimensional, the existing approaches for lumped parameter systems (LPS) are hard to be directly used for control [4]: due to practical limitations, actual distributed control systems are implemented with a finite number of actuators and sensors. Hence, designing such finite-dimensional control systems with guaranteed performance becomes a challenging task. In this way, several approaches have been proposed for

J.L. Pitarch is with the DPT of Systems Engineering and Automatic Control, EII, Universidad de Valladolid. C/Real de Burgos S/N, 47011, Valladolid, SPAIN. Corresponding author. e-mail: jose.pitarch@autom.uva.es

M. Rakhshan is with the DPT of Electrical Engineering, University of Notre Dame, Notre Dame, IN 46556, USA. e-mail: mrakhsha@nd.edu

M.M. Mardani and M. Shasadeghi are with the DPT of Electrical and Electronics Engineering, Shiraz University of Technology, Modarres Blv., Shiraz, IRAN. e-mail: m.mardani@sutech.ac.ir, shasadeghi@sutech.ac.ir the control synthesis of PDE systems. In general, these methods fall into two types: "indirect" and "direct" [5]. Indirect ones employ the infinite-dimensional PDE model to design the controller, which is then lumped for implementation aim [1]. Direct approaches apply a spatial discretization (finite differences, orthogonal collocation or Galerkin) to the PDE system in order to obtain an approximate model that contains a set of ordinary differential equations (ODEs) in time [6]. The subsequent approximate ODE model is then used to design finite-dimensional controllers.

Stabilization of hyperbolic PDE systems in industry has been done typically using PIDs and boundary control [7], [8]. However, as PID designs are normally based on linearized models, finding the right tuning in order to provide good robustness/performance tradeoffs is a difficult task [9]. Optimization-based approaches have been also presented in [10], [11] to deal with such issue, where linear quadratic (LQ) optimal regulators were proposed for a class of hyperbolic PDE systems via spectral factorization. Nevertheless, these linear designs do not give a priori theoretical guarantees of performance and constraint satisfaction in all the desired region of operation.

Such theoretical guarantees for PDE systems can be obtained via the extension of the Lyapunov's second method to infinite dimensional systems. This method requires the construction of a Lyapunov Functional (LF) [12] but a priori choices for LF structures are difficult to make. In this way, several researches have been carried out along the last decade to overcome this issue: locally guaranteed control of nonlinear parabolic PDE systems [13] as well as hyperbolic ones [14] have been proposed via fuzzy TS approaches, using the wellknown systematic sector nonlinearity modeling and semidefinite programming. In particular, since the seminal paper [14] appeared, the $\mathcal{L}_{2}$-norm stabilization of semilinear first-order hyperbolic PDE systems using spatial differential/algebraic LMIs has been extended. Hence, on the basis of the existing SDLMI technique, [4] addressed the distributed $\mathcal{H}_{\infty}$ control considering input constraints, [15] proposed an output feedback based on distributed-fuzzy observers, [16] addressed the tracking problem with variable-structure controllers ensuring exponential or practical stability and, recently, the static $\mathcal{H}_{\infty}$ output feedback with Markovian actuator faults was presented in [17]. Furthermore, the quadratic framework was left in [18] using fuzzy Lyapunov functions.

Moreover, the SOS programming tools [19] developed for the analysis and control of polynomial ODE systems [20], [21], [22] are also useful to analyze PDE systems: stability for some classes of PDE systems is checked via polynomial 
LFs in [23], [12]. However, such results only deal with PDE systems of pure polynomial nonlinearities. In addition, there is a common drawback in many of the above referred TS or polynomial control designs: the actuators' physical limitations are sometimes neglected or their treatment leads to very conservative controllers, despite considering them is mandatory for both performance guarantees in practice and adequate equipment sizing. Therefore, we consider that treating them appropriately from the design phase is of key importance.

Also recently, TS approaches for ODE systems have been extended to use polynomial vertex models instead of linear ones [24]. If the Taylor series approach [25] is used to compute such fuzzy-polynomial models, they allow to asymptotically reduce the conservatism in stability analysis when using shape-independent constraints. Thereupon, SOS tools are also able to obtain numerical global or local solutions for fuzzypolynomial systems [26], [27]. There are, of course, other model-based control strategies which explicitly consider input and/or state constraints [28], but they require the execution of online optimization routines, so they have been intentionally left out of the paper's scope.

Focusing on semilinear hyperbolic PDE systems with Dirichlet boundary condition, the aim of this paper is finding a distributed nonlinear state-feedback law which gives a control profile ensuring performance and input-constraint satisfaction in practice. The proposed synthesis approach combines fuzzypolynomial modeling [25], Positivstellensatz [29] and SOS programming to derive a set of Lyapunov constraints ensuring exponential stability of the closed-loop system in a local region of initial conditions. Briefly, the rest of this paper organizes as follows: Section $\mathrm{II}$ recalls some preliminary results; Section III introduces the fuzzy-polynomial PDE model and gives the problem statement; the main result on the control synthesis is given in Section IV, the effectiveness of the approach is tested in Section V] with the typical example of a nonisothermal PFR; last, Section VI gathers some conclusions and draws possible future research lines.

Notation: $I$ stands for the identity matrix of suitable dimensions. Unless otherwise stated, $\|\cdot\|$ will state the Frobenius norm. A positive definite symmetric matrix $M$ is denoted by $M \succ 0$ ( $M \succeq 0$ stands for positive semidefinite). A symmetric matrix $P(x)$ in the spatial variable $x$ is positive definite (positive semidefinite) in an interval $l_{1} \leq x \leq l_{2}$ if $P(x) \succ 0$ $(P(x) \succeq 0)$ for all $x \in\left[l_{1}, l_{2}\right]$. The symbol $(*)$ is used to denote the symmetric element in matrix expressions, e.g., $[M(x)+N(x)+(*)] \equiv\left[M(x)+N(x)+M^{T}(x)+N^{T}(x)\right] . \mathrm{A}$ SOS polynomial $p(y)$ in variables $y$ is denoted by $p(y) \in \Sigma_{y}$. Similarly, an $n \times m$ SOS polynomial matrix $L(y)$ will be denoted by $L(y) \in \Sigma_{y}^{n \times m}$. $\operatorname{Proj}_{x}((x, w)):=x$ will project on $x$ coordinate. Finally, $\mathcal{H}$ denotes the infinite-dimensional Hilbert space of $N$-dimensional square-integrable vector functions defined on an interval $\left[l_{1}, l_{2}\right]$, and $\mathcal{L}^{1}(\mathcal{H})$ the space of Lebesgue integrable functions on $\mathcal{H}$.

\section{PRELIMINARIES}

This section summarizes well-known results which be useful throughout the paper.
Proposition 1. Assume an invertible matrix $P(x)$. Hence, $P(x)^{-1} P(x)=I$. Differentiating both sides with respect to $x$ yields:

$$
\frac{\partial P(x)^{-1}}{\partial x} P(x)+P(x)^{-1} \frac{\partial P(x)}{\partial x}=0
$$

Thus, the following relation holds:

$$
P(x)^{-1} \frac{\partial P(x)}{\partial x} P(x)^{-1}=-\frac{\partial P(x)^{-1}}{\partial x}
$$

Consider an affine-in-control nonlinear system formed by $n$ ordinary differential equations

$$
\dot{y}(t)=f(y(t))+B(y(t)) u(t)
$$

where $y(t) \in \mathbb{R}^{n}$ and $u(t) \in \mathbb{R}^{m}$ are the state and control vectors, respectively. Furthermore, $f(\cdot)$ and $B(\cdot)$ may be vectors of Lipschitz continuous nonlinear functions with suitable dimensions.

Local fuzzy-polynomial modeling: Consider a compact region of the state space $\Omega(y(t)) \subset \mathbb{R}^{n}$. Results in [25] state that any sufficiently smooth function of one real variable $f(y(\cdot))$, such that its Taylor expansion exists, can be equivalently represented in $\Omega$ by a fuzzy model (linear or polynomial) using sector conditions. This methodology can be also applied to any function that can be written as an expression tree with functions of one variable, i.e., addition and multiplication.

Definition 1 ([25]). A local fuzzy-polynomial model to exactly represent the system (3) in a compact region $\Omega$ can be obtained via Taylor series as

$$
\dot{y}(t)=\sum_{i=1}^{r} \mu_{i}(z)\left(p_{i}(y(t))+B_{i}(y(t)) u(t)\right)
$$

where $z$ are known or measurable quantities (states, external inputs and/or time), $p_{i}(y(t)) \in \mathcal{R}_{y}^{n}$ and $B_{i}(y(t)) \in \mathcal{R}_{y}^{n \times m}$ are the $r$ polynomial consequent model ${ }^{1}$, being $\mu_{i}(z)$ their corresponding nonlinear membership functions which belong to the $(r-1)$-dimensional standard simplex:

$$
\Gamma=\left\{\mu \in \mathbb{R}^{r}: \sum_{i=1}^{r} \mu_{i}=1, \quad \mu_{i} \geq 0\right\}
$$

Remark 1. The modeling methodology in [25] allows to select a maximum degree for the polynomial consequent models. This choice defines a tradeoff between complexity and conservatism: the higher the degree is chosen, the closer the models are to the original nonlinear system but, the more computational resources will be required too. Indeed, the Taylor series of degree one ends up in the well-known TS or linear-parameter varying (LPV) case. Note also that the size of the modeling region influences the conservativeness [30]. Usually the smaller the set $\Omega$ is chosen, the closer the consequent models can be for the same degree. In particular, if $\Omega$ is a small enough neighbourhood of the origin, the consequent models will tend to the Taylor series.

\footnotetext{
${ }^{1}$ Obtained by cutting the Taylor series up to a desired degree and computing bounds for the Taylor's reminder in the region $\Omega$. See [25] for further details and examples.
} 
Remark 2. The reduction of conservatism by increasing the degree of polynomial consequent models may be not true for smooth non-analytic functions, if the Taylor series does not equal $f(y(\cdot)) \forall y(\cdot) \in \Omega$, e.g., the Taylor series of $\log (1+y(\cdot))$ is only a good approximation in the range $-1<y(\cdot) \leq 1$.

Copositive Summation Problem: In closed loop with a fuzzy state-feedback control law $u(t)=\sum_{i=1}^{r} \mu_{i}(z) k_{i}(y(t))$, $k_{i}(y(t)) \in \mathcal{R}_{y}^{m}$, the dynamics of (4) becomes

$$
\dot{y}(t)=\sum_{i=1}^{r} \sum_{j=1}^{r} \mu_{i}(z) \mu_{j}(z)\left(p_{i}(y(t))+B_{i}(y(t)) k_{j}(y(t))\right)
$$

where double summations on the standard simplex appear. These double summations must be subsequently translated to Lyapunov-decrease conditions, for instance, in the form:

$$
\Xi(\mu, z, y, t)=\sum_{i=1}^{r} \sum_{j=1}^{r} \mu_{i}(z) \mu_{j}(z) \Xi_{i j}(y(t)) \leq 0 \quad \forall t \geq 0
$$

Widely-known sufficient conditions for (6) are reported in the literature [31], [32], [33].

To ease further notation, the time $t$ is omitted for the rest of the section. Hence, notation $\operatorname{COP}_{\mu}[\Xi(y) \leq 0]$ will denote any semialgebraic set $\hat{\Xi} \subset\{y: \Xi(\mu, z, y) \leq 0 \forall \mu \in \Gamma\}$, obtained via any shape-independent (replacing $\mu_{i}, \mu_{j}$ by arbitrary scalars in $\Gamma$ ) sufficient relaxation of the copositive problem (expressed as polynomial inequalities). These relaxations may range from the naive $\hat{\Xi}=\left\{y: \Xi_{i j}(y)+\Xi_{j i}(y) \in \Sigma_{y}, \forall i \leq j\right\}$, to the asymptotically exact ones based on the coefficients of $\left(\sum_{i} \mu_{i}\right)^{d} \Xi \in \Sigma_{\mu, y}$ in [32], [34].

SOS programming: The general SOS problem is checking the non-negativity of a polynomial $p(y)$ in variables $y$, by the conservative condition of $p(y)$ being SOS. Such SOS decompositions of polynomials can be found using semidefinite programming (SDP) [19]. The classical LMI framework can be also extended to polynomial cases by checking if a polynomial matrix $L(y)$ is positive semi-definite for all $y$, i.e., $L(y)$ is SOS if the polynomial $v^{T} L(y) v \in \Sigma_{y, v}$. See [35] for further details.

Consider now a region $\Omega$ defined by $g_{i}(y), h_{j}(y)$ polynomial boundaries as follows:

$$
\begin{aligned}
\Omega:=\left\{y: g_{1}(y)>0, \ldots, g_{n_{g}}(y)\right. & >0 \\
h_{1}(y) & \left.=0, \ldots, h_{n_{h}}(y)=0\right\}
\end{aligned}
$$

Local positivity of polynomials in $\Omega$ can be checked via the well-known Positivstellensatz theorem [29]. The following lemma is reduced version of such theorem (used in later in the example):

Lemma 1. If polynomials $s_{i}(y) \in \Sigma_{y}$ and $r_{j}(y) \in \mathcal{R}_{y}$ can be found fulfilling

$$
p(y)-\epsilon(y)-\sum_{i=1}^{n_{g}} s_{i}(y) g_{i}(y)+\sum_{j=1}^{n_{h}} r_{j}(y) h_{j}(y) \in \Sigma_{y}
$$

then $p(y)$ is locally greater or equal than $\epsilon(y)$ in $\Omega$.

Based also in Positivstellensatz results, we can set SOS conditions for a semialgebraic set $Z=\left\{z_{k}(y)>0, k=\right.$ $\left.1, \ldots, k_{M}\right\}$ to be included in $\Omega$. For instance, the existence of multipliers $\left(s_{i}, s_{i k}\right) \in \Sigma_{y}, r_{i j} \in \mathcal{R}_{y}$ such that $s_{i} g_{i}+\sum_{j}^{n_{h}} r_{i j} h_{j}-\sum_{k=1}^{k_{M}} s_{i k} z_{k} \in \Sigma_{y}, i: 1, \ldots, n_{g}$. Such conditions (or any other sufficient SOS relaxation) will be shorthanded by $\mathcal{P} \mathcal{S}[Z \subset \Omega]$. Conditions in $(8)$ will be a particular SOS implementation choice of a sufficient condition for $\Omega \subset\{y: p(y)-\epsilon(y) \geq 0\}$, symbolized by $\mathcal{P S}[\Omega \subset\{y: p(y)-\epsilon(y) \geq 0\}]$. Also, abusing the notation, $\mathcal{P S}[\Omega \subset\{y: L(y) \succeq 0\}]$ will denote any sufficient SOS condition for $\Omega \subset\{y: L(y) \succeq 0\}$ being $L(y) \in \mathcal{R}_{y}^{l \times l}$, such as $v^{T}\left[L(y)-\sum_{i} s_{i}(y) g_{i}(y) I+\sum_{j} r_{j}(y) h_{j}(y) I\right] v \in \Sigma_{y, v}^{l}$. In this way, many analysis and control problems within the polynomial framework can be handled by combining the SOS optimization tools with the Positivstellensatz theorem, e.g., [21], [22], [12], [36].

\section{Problem Statement}

We first recall some stability properties for the class of PDE systems under consideration. Consider the semilinear firstorder hyperbolic PDE systems in one spatial dimension $x$ with the following state-space description

$$
\frac{\partial y(x, t)}{\partial t}=\Theta \frac{\partial y(x, t)}{\partial x}+f(y(x, t), x)+B(y(x, t), x) u(x, t)
$$

together with the Dirichlet boundary condition and the initial condition:

$$
y\left(l_{1}, t\right)=0 \quad y(x, 0)=y_{0}(x)
$$

Here $y(x, t)=\left[y_{1}(x, t) \ldots y_{n}(x, t)\right]^{T} \in \mathcal{L}_{2}\left(\left[l_{1}, l_{2}\right] ; \mathbb{R}^{n}\right)$ indicates the state vector, $x \in\left[l_{1}, l_{2}\right] \subset \mathbb{R}$ and $t \in[0, \infty)$ are the spatial position and time, respectively. Consequently, $u(x, t)=$ $\left[u_{1}(x, t), \ldots, u_{m}(x, t)\right]^{T} \in \mathcal{L}_{2}\left(\left[l_{1}, l_{2}\right] ; \mathbb{R}^{m}\right)$ indicates the control input vector and $f(y, \cdot), B(y, \cdot)$ are matrices of appropriate dimensions whose entries may be locally Lipschitz continuous nonlinear functions in the states $y$. Further, $f(0, x)=0$ for all $x \in\left[l_{1}, l_{2}\right]$. The spatially-dependent trajectories of system (9) starting from $y_{0}(x)$ will be denoted by $\psi\left(t, y_{0}(x)\right)$.

Assumption 1. The matrix $\Theta \in \mathbb{R}^{n \times n}$ is diagonal and takes the form $\Theta=\alpha I, \alpha \leq 0$.

The model (9) includes the spatially-invariant case $f(y)$, $g(y)$, which describes many convection-reaction processes that arise in chemical engineering. Also, 9 is assumed distributed affine in control because it is a common configuration in practice, such as applications where the jacket temperature is chosen as the manipulated variable [1].

Let $\omega_{1}$ and $\omega_{2}$ be two elements of $\mathcal{H}$. Then the inner product and the norm in $\mathcal{H}$ are defined by:

$$
\begin{aligned}
\left\langle\omega_{1}, \omega_{2}\right\rangle & =\int_{l_{2}}^{l_{1}}\left\langle\omega_{1}(x), \omega_{2}(x)\right\rangle_{\mathbb{R}^{\mathbb{N}}} d x \\
\left\|\omega_{1}\right\|_{2} & =\sqrt{\left\langle\omega_{1}, \omega_{1}\right\rangle}
\end{aligned}
$$

Where $\left\langle\omega_{1}(x), \omega_{2}(x)\right\rangle_{\mathbb{R}^{\mathbb{N}}}$ denotes the inner product in the Euclidean space $\mathbb{R}^{N}$.

Define $\chi(\cdot, t)$ and $\tilde{u}(\cdot, t)$ to be continuous state and input functions on $\mathcal{H}$ respectively as $\chi(\cdot, t):=y(x, t)$ and $\tilde{u}(\cdot, t):=$ 
$u(x, t)$ for $t>0, x \in\left[l_{1}, l_{2}\right]$. Define the spatial differential operator $\mathcal{A}$ in $\mathcal{H}$ and its domain by:

$$
\begin{gathered}
\mathcal{A}:=\Theta \frac{\partial}{\partial x} \\
\mathcal{D}(\mathcal{A})=\left\{\chi \in \mathcal{H}, \frac{\partial \chi}{\partial x} \in \mathcal{H}, \chi(\cdot, 0)=\chi_{0}, \chi\left(l_{1}, t\right)=0\right\}
\end{gathered}
$$

Then 9 can be rewritten as the following differential equation on the Hilbert space ([14] and references therein)

$$
\dot{\chi}(\cdot, t)=\mathcal{A} \chi(\cdot, t)+\tilde{f}(\chi(\cdot, t))+\tilde{B}(\chi(\cdot, t)) \tilde{u}(\cdot, t), t \geq 0, \chi(\cdot
$$

being $\tilde{f}(\chi):=f(y, x)$ and $\tilde{B}(\chi):=B(y, x)$.

The infinitesimal operator $\mathcal{A}$ generates an exponentially stable $C_{0}$-semigroup $\mathcal{T}(t)$ on $\mathcal{H}$ whose induced norm satisfies $\|\mathcal{T}(t)\|_{2} \leq \sigma e^{-\varpi t}$ with $\sigma>0$ and $\varpi>0$. Then, the local existence of a unique classical solution to (12) can be proven with $\tilde{u}(t) \equiv 0$. Also, if $\tilde{u} \in \mathcal{L}^{1}(0, \tau: \mathcal{H})$ is continuous, there exist a generalized mild solution for every $\chi \in \mathcal{D}(\mathcal{A})$. Moreover, if $\tilde{u} \in \mathcal{D}(\mathcal{A})$ and $\mathcal{A} \tilde{u}(t) \in \mathcal{L}^{1}(0, \tau: \mathcal{H})$, such mild solution becomes a classical one [37].

Remark 3. Local solutions in time, e.g., finite-escape time, will be excluded with the Lyapunov-based conditions in Section IV From now on, all mention to locality will refer to subsets of the state space.

Next we introduce the concept of exponential stability used for the later developments.

Definition 2. The system (9) with $u(x, t) \equiv 0$ is said exponentially stable with decay rate $\rho>0$, if there exists $\kappa>0$ such that:

$$
\|\widehat{y}(y(x, t))\|_{2}^{2} \leq \kappa e^{-\rho t}\left\|\widehat{y}\left(y_{0}(x)\right)\right\|_{2}^{2} \quad \forall t \geq 0
$$

The term $\widehat{y}(y(x, t)) \in \mathcal{R}^{N}$ is a column vector whose entries are monomials in $y(x, t)$ belonging to $\mathcal{H}$. A monomial in $y$ is a function with form $y_{1}^{\alpha_{1}} y_{2}^{\alpha_{2}} \cdots y_{n}^{\alpha_{n}}$, where $\alpha_{1}, \alpha_{2}, \cdots, \alpha_{n}$ are nonnegative integers. It is assumed that $\widehat{y}(y(x, t))=0$ iff $y(x, t)=0$.

In the following, notations of time $t$ and spatial variable $x$ are omitted when no confusion arises, e.g., $\widehat{y}(y(x, t))$ is stated as $\widehat{y}(y)$. Now, consider a local region of the state space $\Omega$, defined by $g_{i}(y(x, \cdot)), h_{j}(y(x, \cdot))$ polynomial boundaries:

$$
\begin{aligned}
\Omega:=\{y(x, \cdot) & \in \mathcal{H}, x \in\left[l_{1}, l_{2}\right]: g_{i}(y(x, \cdot))>0, \\
& \left.i: 1, \ldots, n_{g}, h_{j}(y(x, \cdot))=0, j: 1, \ldots, n_{h}\right\}
\end{aligned}
$$

Definition 3. The Local Domain of Attraction (LDA) of system (9) with $u(x, t) \equiv 0$, referred to region (14) and denoted by $\mathcal{D}_{\Omega}$, is defined as the set of initial conditions fulfilling:

$$
\mathcal{D}_{\Omega}:=\left\{y_{0}(x) \in \Omega: \begin{array}{c}
\psi\left(t, y_{0}(x)\right) \in \Omega \forall t \geq 0 \\
\lim _{t \rightarrow \infty} \psi\left(t, y_{0}(x)\right)=0
\end{array}\right\}
$$

Fuzzy-polynomial PDE modeling: Using the Taylor series approach, the semilinear hyperbolic PDE system (9) can be exactly described in the region (14) by the following fuzzypolynomial PDE model

$$
\begin{array}{r}
\frac{\partial y(x, t)}{\partial t}=\Theta \frac{\partial y(x, t)}{\partial x}+\sum_{i=1}^{r} \mu_{i}(z(x, t))\left[A_{i}(y(x, t), x)\right. \\
\left.\widehat{y}(y(x, t))+B_{i}(y(x, t), x) u(x, t)\right]
\end{array}
$$

where $A_{i}(y(x, t), x) \in \mathcal{R}^{n \times N}, B_{i}(y(x, t), x) \in \mathcal{R}^{n \times m}$ are polynomial matrices with suitable dimensions and $z(x, t)$ are measurable space-time varying quantities. In the following, it is assumed that $\mu_{i}(z(x, t)) \geq 0$ and $\sum_{i=1}^{r} \mu_{i}(z(x, t))=1$ for all $x \in\left[l_{1}, l_{2}\right]$ and $t \geq 0$.

Based on the model (16), consider the subsequent fuzzy state-feedback law with saturation for controlling the nonlinear PDE system (9)

$$
\begin{aligned}
u(x, t) & =\operatorname{sat}(\nu(x, t)) \\
\nu(x, t) & =\sum_{j=1}^{r} \mu_{j}(z(x, t)) K_{j}(y(x, t), x) \widehat{y}(y(x, t))
\end{aligned}
$$

where the to be computed controller gains $K_{j}(y, x)=$ $M_{j}(y, x) P^{-1}(y(x, t), x), M_{j}(y, x) \in \mathcal{R}_{y, x}^{m \times N}, P(y(x, t), x) \in$ $\mathcal{R}_{y, x}^{N \times N}$, are defined in $x \in\left[l_{1}, l_{2}\right]$. Note that these controller gains $K_{j}$ are neither restricted to be constant nor polynomial, but may be rational in the state variables. This fact, together with the nonlinear nature of the membership functions $\mu_{j}$, gives great freedom to get complex nonlinear controllers.

The control law (17) must fulfill a componentwise saturation constraint:

$$
\begin{aligned}
\left|u^{[k]}(x, t)\right| & \leq \eta_{k} \quad \forall(y, x) \in \Omega \\
\operatorname{sat}\left(\nu^{[k]}(x, t)\right) & :=\operatorname{sign}\left(\nu^{[k]}(x, t)\right) \min \left(\left|\nu^{[k]}(x, t)\right|, \eta_{k}\right)
\end{aligned}
$$

Here $\eta_{k} \in \mathbb{R}^{+}$and $u^{[k]}$ denotes the $k$-th element of the input vector. Likewise, notation $K_{j}^{[k]}(y, x)$ will denote the $k$-th row of the matrix $K_{j}(y, x)$.

Now, defining $\Psi(\nu):=\nu-\operatorname{sat}(\nu)$, from (16)-(17) the overall closed-loop system can be written as:

$$
\begin{array}{r}
\frac{\partial y(x, t)}{\partial t}=\Theta \frac{\partial y(x, t)}{\partial x}+\sum_{i=1}^{r} \sum_{j=1}^{r} \mu_{i}(z(x, t)) \mu_{j}(z(x, t)) \\
\bar{A}_{i j} \widehat{y}(y(x, t))-B_{i}(y(x, t), x) \Psi(\nu) \\
\bar{A}_{i j}=A_{i}(y(x, t), x)+B_{i}(y(x, t), x) K_{j}(y(x, t), x)
\end{array}
$$

Remark 4. If $\widehat{y}(y(x, t))=y(x, t), A_{i}(y, x)=A_{i}(x)$, $B_{i}(y, x)=B_{i}(x)$ and $K_{j}(y, x)=K_{j}(x)$, then (19) reduces to the classical TS/LPV PDE representation used in the related literature [4], [17].

Hence, the objective of this paper is finding a suitable state feedback controller (17) fulfilling the bounds (18) such that, in closed loop with 9, ensures exponential stability with a decay rate $\rho>0$ starting from initial conditions $y_{0}(x) \subset \Omega(y, x)$. 


\section{Distributed CONTROL SYNTHESIS}

A Lyapunov-based SOS design is proposed next such that the closed-loop system (19) is locally exponentially stable in the sense of definitions 2 and 3 Consider the following candidate Lyapunov functional

$$
V(t)=\int_{l_{1}}^{l_{2}} \widehat{y}^{T}(y(x, t)) P^{-1}(y(x, t), x) \widehat{y}(y(x, t)) d x,
$$

where $P(y(x, t), x)=P^{T}(y(x, t), x) \in \mathcal{R}_{y, x}^{N \times N}$ is a positivedefinite polynomial matrix defined in $\Omega$.

Lemma 2. If for the closed-loop system (19) there exists a Lyapunov functional (20) and a scalar $\rho>0$ satisfying

$$
\frac{d V(t)}{d t}+\rho V(t) \leq 0 \forall t \geq 0, \quad(y, x) \in \Omega,
$$

then exponential stability in the sense of Definition 2 is achieved locally in $\Omega$ and there exists $c \in \mathbb{R}^{+}$such that the set $V_{c}:=\left\{y_{0}(x): \widehat{y}^{T}\left(y_{0}(x)\right) P^{-1}\left(y_{0}(x), x\right) \widehat{y}\left(y_{0}(x)\right) \leq c\right\} \subset \Omega$ belongs to the LDA of (19).

Proof. Integrating (21) with respect to $t$ we obtain:

$$
V(t) \leq V(0) e^{-\rho t}
$$

Now, as $P^{-1}(y, x)$ is a spatially-continuous positive matrix function in $\Omega$, it has bounded minimum and maximum eigenvalues $\lambda\left(P^{-1}(y, x)\right)>0$ for $(y, x) \in \Omega$. Then, $V(t)$ satisfies

$$
a\|\widehat{y}(y(x, t))\|_{2}^{2} \leq V(t) \leq b\|\widehat{y}(y(x, t))\|_{2}^{2}
$$

with $a:=\min _{i \in\{1, \ldots, n\}}\left\{\min _{(y, x) \in \Omega}\left\{\lambda_{i}\left(P^{-1}(y, x)\right)\right\}\right\}$ and $b:=\max _{i \in\{1, \ldots, n\}}\left\{\max _{(y, x) \in \Omega}\left\{\lambda_{i}\left(P^{-1}(y, x)\right)\right\}\right\}$. Then, using 22, we can write

$$
\begin{aligned}
a \int_{l_{1}}^{l_{2}}\|\widehat{y}(y(x, t))\|^{2} d x \leq V(t) & \leq \\
V(0) e^{-\rho t} & \leq e^{-\rho t} b \int_{l_{1}}^{l_{2}}\left\|\widehat{y}\left(y_{0}(x)\right)\right\|^{2} d x
\end{aligned}
$$

for initial conditions $y_{0}(x) \in \mathcal{D}_{\Omega}$. So, taking $\kappa=b / a$, it implies that (13) in Definition 2 holds locally in $\Omega$. In such case, by Lyapunov stability theory, 201-21] ensure that a trajectory $\psi\left(t, y_{0}(x)\right)$ which crosses a Lyapunov level set $V_{c}:=\left\{(y, x): \widehat{y}^{T}(y) P^{-1}(y, x) \widehat{y}(y) \leq c\right\}$ never can come out again from it. Thus, each $c>0$ such that $V_{c} \subset \Omega$ is an estimate of the LDA for system (19).

Let $w \in \mathbb{R}^{m}$ and $\sigma \in \mathbb{R}^{N}$. Then, the set $\Pi$ is defined by quadruplets of variables $y, x, w, \sigma$ as

$$
\begin{aligned}
\Pi:=\{(y, x, w, \sigma) & :(y, x) \in \Omega, \\
& \left.\eta_{k}^{2}-\left(M_{j}^{[k]}(y, x) \sigma-w^{[k]}\right)^{2} \geq 0\right\}
\end{aligned}
$$

for all $j: 1, \ldots, r$ and $k: 1, \ldots, m$.

Theorem 1. If there exist polynomial matrices $P(y, x)$, $M_{j}(y, x)$, scalars $(\rho, \eta, \epsilon)>0$ and Positivstellensatz multipliers fulfilling:

$$
\mathcal{P S}[\Omega \subset\{y, x: P(y, x)-\epsilon I \succeq 0\}]
$$

$$
\begin{gathered}
\mathcal{P} \mathcal{S}\left[\Pi \subset C O P _ { \mu } \left[\sigma ^ { T } \left(\Theta \frac{\partial P(y, x)}{\partial x}+\Xi(y, x)+\right.\right.\right. \\
\left.\left.\rho P(y, x)) \sigma-2 w^{T} B_{i}^{T}(y, x) \sigma \leq 0\right]\right] \\
\mathcal{P} \mathcal{S}\left[\left\{y, x: P(y, x)-\widehat{y} \cdot \widehat{y}^{T} \succeq 0\right\} \subset \operatorname{Proj}_{y, x}(\Pi)\right] \\
\mathcal{P} \mathcal{S}\left[\left\{x: P\left(y_{0}, x\right)-\widehat{y}\left(y_{0}\right) \cdot \widehat{y}^{T}\left(y_{0}\right) \succeq 0\right\}\right. \\
\left.\subset\left\{x: l_{2}-x \geq 0, x-l_{1} \geq 0\right\}\right] \\
\Xi(y, x)=\sum_{i=1}^{r} \sum_{j=1}^{r} \mu_{i}(y) \mu_{j}(y) \cdot\left(T ( y ) \left(A_{i}(y, x) P(y, x)+\right.\right. \\
\left.B_{i}(y, x) M_{j}(y, x)\right)+(*)-\sum_{k=1}^{n} \frac{\partial P(y, x)}{\partial y{ }^{[k]}}\left(\left(\left(A_{i}(y, x)\right.\right.\right. \\
\left.\left.\left.P(y, x))^{[k]}+B_{i}^{[k]}(y, x) M_{j}(y, x)\right) \sigma-B_{i}^{[k]}(y, x) w\right)\right)
\end{gathered}
$$

where $T(y)=\frac{\partial \widehat{y}(y)}{\partial y} \in \mathcal{R}_{y}^{N \times n}$, then the closed-loop system (19) is locally exponentially stable with decay rate $\rho$ in the region (14), with controller (17) fulfilling bounds (18). The controller gains can be obtained as $K_{j}(y, x)=$ $M_{j}(y, x) P^{-1}(y, x)$.

Proof. Condition 24] ensures $V(t)>0 \forall(y, x) \in\{\Omega / y=$ $0\}$, which can be checked by performing the standard change of variable $\widehat{y}=P(y, x) \sigma$ in $\widehat{y}^{T} P^{-1}(y, x) \widehat{y}$, leading to search for a $P(y, x) \succ 0$ in $\Omega$. Condition (26) ensures the level set $\left\{(y, x): \widehat{y}^{T} P^{-1}(y, x) \widehat{y} \leq 1\right\}$ belongs to the region $\Pi$ by applying Schur complement twice:

$$
\begin{aligned}
1-\widehat{y}^{T} P^{-1}(y, x) \widehat{y} \geq 0 \Leftrightarrow\left(\begin{array}{cc}
1 & \widehat{y}^{T} \\
\widehat{y} & P(y, x)
\end{array}\right) \succeq 0 \\
\quad \Leftrightarrow P(y, x)-\widehat{y} \cdot \widehat{y}^{T} \succeq 0
\end{aligned}
$$

Hence, undoing the variable changes $M_{j}(y, x)=$ $K_{j}(y, x) P(y, x), \sigma=P^{-1}(y, x) \widehat{y}$ and $w=\Psi(\nu)$ in (23), 26) is ensuring that the control bound (18) holds in $\left\{(y, x): \widehat{y}^{T} P^{-1}(y, x) \widehat{y} \leq 1\right\} \subset \Omega$. Following an analogous development, condition (27) is ensuring the initial conditions $y_{0}(x)$ belong to the above referred level set. Finally we will prove that 25) ensures 21] in the region $\Pi$, i.e., it ensures (21) with the bound (18). Recalling the Lyapunov functional (20) and the closed-loop system (19), the stability condition (21) yields:

$$
\begin{gathered}
\frac{d V(t)}{d t}+\rho V(t)=\int_{l_{1}}^{l_{2}}\left(\widehat{y}^{T}(y) P^{-1}(y, x) \frac{d \widehat{y}(y)}{d t}+(*)+\right. \\
\left.\widehat{y}^{T}(y) \frac{d P^{-1}(y, x)}{d t} \widehat{y}(y)\right) d x+\rho \int_{l_{1}}^{l_{2}} \widehat{y}^{T}(y) P^{-1}(y, x) \widehat{y}(y) d x= \\
\int_{l_{1}}^{l_{2}}\left(\left(\widehat { y } ^ { T } ( y ) P ^ { - 1 } ( y , x ) T ( y ) \left(\Theta \frac{\partial y}{\partial x}+\sum_{i=1}^{r} \sum_{j=1}^{r} \mu_{i}(z) \mu_{j}(z)\right.\right.\right. \\
\left.\left.\left(\bar{A}_{i j} \widehat{y}(y)-B_{i}(y, x) \Psi(\nu)\right)\right)+(*)+\widehat{y}^{T}(y) \frac{d P^{-1}(y, x)}{d t} \widehat{y}(y)\right) d x \\
+\rho \int_{l_{1}}^{l_{2}} \widehat{y}^{T}(y) P^{-1}(y, x) \widehat{y}(y) d x
\end{gathered}
$$


As $\Theta=\alpha I$ by assumption, we can rewrite the term:

$$
\begin{aligned}
\int_{l_{1}}^{l_{2}} \widehat{y}^{T}(y) P^{-1}(y, x) T(y) \Theta \frac{\partial y}{\partial x} d x= \\
\int_{l_{1}}^{l_{2}} \alpha \widehat{y}^{T}(y) P^{-1}(y, x) T(y) \frac{\partial y}{\partial x} d x
\end{aligned}
$$

Then, integrating 29 by parts with the boundary and initial conditions (10), and using Proposition 11, we get

$$
\begin{gathered}
\int_{l_{1}}^{l_{2}} \alpha \widehat{y}^{T}(y) P^{-1}(y, x) \frac{\partial \widehat{y}(y)}{\partial x} d x=\alpha \widehat{y}^{T}\left(y\left(l_{2}, t\right)\right) P^{-1}\left(y, l_{2}\right) \\
\widehat{y}\left(y\left(l_{2}, t\right)\right)-\int_{l_{1}}^{l_{2}} \frac{\partial}{\partial x}\left(\alpha \widehat{y}^{T}(y) P^{-1}(y, x)\right) \widehat{y}(y) d x \\
-\int_{l_{1}}^{l_{2}} \frac{\partial}{\partial x}\left(\alpha \widehat{y}^{T}(y) P^{-1}(y, x)\right) \widehat{y}(y) d x=-\int_{l_{1}}^{l_{2}}\left(\frac{\partial \widehat{y}(y)}{\partial x}\right)^{T} \leq \\
\alpha P^{-1}(y, x) \widehat{y}(y) d x-\int_{l_{1}}^{l_{2}} \widehat{y}^{T}(y) \alpha \frac{d P^{-1}(y, x)}{d x} \widehat{y}(y) d x \\
=-\int_{l_{1}}^{l_{2}}\left(\frac{\partial \widehat{y}(y)}{\partial x}\right)^{T} \alpha P^{-1}(y, x) \widehat{y}(y) d x+ \\
\int_{l_{1}}^{l_{2}} \widehat{y}^{T}(y) \alpha P^{-1}(y, x) \frac{d P(y, x)}{d x} P^{-1}(y, x) \widehat{y}(y) d x
\end{gathered}
$$

which implies the following inequality:

$$
\begin{aligned}
\int_{l_{1}}^{l_{2}}\left(\widehat{y}^{T}(y) \alpha P^{-1}(y, x) \frac{\partial \widehat{y}(y)}{\partial x}+(*)\right) d x \leq \\
\int_{l_{1}}^{l_{2}} \alpha \widehat{y}^{T}(y) P^{-1}(y, x) \frac{d P(y, x)}{d x} P^{-1}(y, x) \widehat{y}(y) d x
\end{aligned}
$$

Substitution of (31) into 28) results in the bound:

$$
\begin{aligned}
& \frac{d V(t)}{d t}+\rho V(t) \leq \\
& \int_{l_{1}}^{l_{2}}\left(\widehat { y } ^ { T } ( y ) P ^ { - 1 } ( y , x ) T ( y ) \sum _ { i = 1 } ^ { r } \sum _ { j = 1 } ^ { r } \mu _ { i } ( z ) \mu _ { j } ( z ) \left(\bar{A}_{i j} \widehat{y}(y)-\right.\right. \\
& \left.\left.B_{i}(y, x) \Psi(\nu)\right)\right)+(*)+\widehat{y}^{T}(y)\left(\alpha P^{-1}(y, x) \frac{d P(y, x)}{d x} P^{-1}(y, x)\right. \\
& \left.\left.+\rho P^{-1}(y, x)+\frac{d P^{-1}(y, x)}{d t}\right) \widehat{y}(y)\right) d x
\end{aligned}
$$

Hence, using again Proposition 1 and performing $\widehat{y}=$ $P(y, x) \sigma$ in 232, it results in that 21) is satisfied if the following inequality holds:

$$
\begin{gathered}
\sigma^{T}\left(\sum_{i=1}^{r} \sum_{j=1}^{r} \mu_{i}(z) \mu_{j}(z) T(y)\left(\bar{A}_{i j} P(y, x)-B_{i}(y, x) \Psi(\nu)\right)\right. \\
\left.+(*)+\alpha \frac{d P(y, x)}{d x}+\rho P(y, x)-\frac{d P(y, x)}{d t}\right) \sigma \leq 0 \quad
\end{gathered}
$$

Also, considering dynamics 16, we can write:

$$
\begin{gathered}
\alpha \frac{d P(y, x)}{d x}-\frac{d P(y, x)}{d t}=\sum_{k=1}^{n}\left(\alpha \frac{\partial P(y, x)}{\partial y^{[k]}} \frac{\partial y^{[k]}}{\partial x}+\right. \\
\left.\alpha \frac{\partial P(y, x)}{\partial x}-\frac{\partial P(y, x)}{\partial y^{[k]}} \frac{\partial y^{[k]}}{\partial t}\right)=\sum_{k=1}^{n}\left(\alpha \frac{\partial P(y, x)}{\partial x}-\frac{\partial P(y, x)}{\partial y^{[k]}}\right. \\
\sum_{i=1}^{r} \sum_{j=1}^{r} \mu_{i}(z) \mu_{j}(z)\left(\bar{A}_{i j}^{[k]} \cdot \widehat{y}(y)-B_{i}^{[k]}(y, x) \Psi(\nu)\right)
\end{gathered}
$$

Hence, replacing 34 in 33 and changing $K_{j}(y, x)=$ $M_{j}(y, x) P^{-1}(y, x), \widehat{y}=P(y, x) \sigma, \Psi(\nu)=w$, yields the SOS-based condition 25].

Remark 5. Note that the spatially-dependent polynomial conditions in Theorem 1 can be directly checked for SOS, with $(y, x, w, \sigma)$ independent variables. Thus, the obtained controller (17) is infinite dimensional, so it must be lumped for practical implementation using Galerkin [38] or any other discretization method. Note also that conditions in Theorem 1 are shape independent, so the set of initial conditions $V_{1}:=\left\{y_{0}(x):{\widehat{y_{0}}}^{T} P^{-1}\left(y_{0}, x\right) \widehat{y_{0}} \leq 1\right\}$ fulfills $\psi\left(t, y_{0}, \mu\right) \in \Omega$ and $\lim _{t \rightarrow \infty} \psi\left(t, y_{0}, \mu\right)=0 \forall \mu \in \Gamma$. Therefore $V_{1} \subset \mathcal{D}_{\Omega}$ and it is understood as a robust estimate of the LDA [27].

Remark 6. Note that no limit cycles or other attractors than the considered equilibrium profile can appear in $\Omega$ if Theorem 1 renders feasible, because strict positivity of Lyapunov inequalities is ensured by the $\epsilon$ tolerance.

Conditions in Theorem 1 may be nonconvex $\left.\right|^{2}$ because of the products $\rho P, \frac{\partial P}{\partial y^{[k]}} B_{i}^{[k]} M_{j}$ and the different Positivstellensatz multipliers which may multiply either $P$ or $M_{j}$. The following result states a particular finite-dimensional approximation of Theorem 1, with a suitable selection of Positivstellensatz multipliers which allow to cast the problem as a convex one, being thus efficiently solved by SOS programming tools. Consider a region $\Omega$ defined as

$$
\Omega:=\left\{(y, x): \widehat{y}^{T} U_{1} \widehat{y} \leq \zeta_{1}^{2}, \ldots, \widehat{y}^{T} U_{n_{g}} \widehat{y} \leq \zeta_{n_{g}}^{2}, x \in\left[l_{1}, l_{2}\right]\right\}
$$

with $\zeta \in \mathbb{R}^{+}$and $U_{j}$ being constant user-defined matrices with suitable dimensions. Denote by $\mathcal{K}:=\left[k_{1}, k_{2}, \ldots, k_{l}\right]$ the subset of indices $k$ such that $B_{i}^{[k]}(y, x)=0 \forall i, k \in \mathcal{K}$ [24]. Assume that the spatial length $x \in\left[l_{1}, l_{2}\right]$ is gridded in $X$ equally-distributed points. Then, define $\tilde{y}:=\left[y_{k_{1}}, y_{k_{2}}, \ldots, y_{k_{l}}\right]$ and $\Omega_{q}:=\Omega\left(y, x_{q}\right)$ with $q \in X, x_{q}=l_{1}+\frac{l_{2}-l_{1}}{X} q$, i.e., $\Omega_{q}$ is the cutting hyperplane of $\Omega$ in $x=x_{q}$.

Theorem 2. Given scalars $(\rho, \eta, \epsilon)>0$, if there exist polynomial matrices $P_{q}(\tilde{y}), M_{q j}(y), L_{q j}(y)$, plus Positivstellensatz multipliers $\mathcal{S}(y) \in \Sigma_{y}^{N \times N}$ and $\vartheta(y) \in \mathcal{R}_{y}^{m \times m}, \vartheta$ being diagonal, fulfilling:

$$
P_{q}(\tilde{y})-\epsilon I-\sum_{z=1}^{n_{g}} \mathcal{S}_{1 q z}(y)\left(\zeta_{z}^{2}-\widehat{y}^{T} U_{z} \widehat{y}\right) \in \Sigma_{y}^{N \times N} q: 0, \ldots, X
$$

\footnotetext{
${ }^{2}$ Only in some very particular cases of linear polytopic systems, quadratic $V(y)$, Theorem 1 reduces to the convex cases in the literature referred in the introduction.
} 


$$
\begin{gathered}
\sigma^{T}\left(\frac{\Theta}{\Delta x}\left(P_{q-1}(\tilde{y})-P_{q}(\tilde{y})\right)-\Xi_{q i i}-\rho P_{q}(\tilde{y})\right. \\
\left.-\sum_{z=1}^{n_{g}} \mathcal{S}_{2 q i z}(y)\left(\zeta_{z}^{2}-\widehat{y}^{T} U_{z} \widehat{y}\right)\right) \sigma+\Upsilon_{q i i} \in \Sigma_{y, \sigma, w} \\
q: 1, \ldots, X ; i: 1, \ldots, r \quad(37) \\
\sigma^{T}\left(\frac{\Theta}{\Delta x}\left(P_{q-1}(\tilde{y})-P_{q}(\tilde{y})\right)-\frac{\Xi_{q i i}}{r-1}-\frac{\Xi_{q i j}+\Xi_{q j i}}{2}-\rho P_{q}(\tilde{y})\right. \\
\left.-\sum_{z=1}^{n_{g}} \mathcal{S}_{2 q i j z}(y)\left(\zeta_{z}^{2}-\widehat{y}^{T} U_{z} \widehat{y}\right)\right) \sigma+\frac{\Upsilon_{q i i}}{r-1}+\frac{\Upsilon_{q i j}+\Upsilon_{q j i}}{2} \\
\in \Sigma_{y, \sigma, w} q: 1, \ldots, X ; i, j: 1, \ldots, r ; i \neq j \quad(38) \\
\zeta_{j}^{2} U_{j}^{-1}-P_{q}(\tilde{y})-\sum_{z=1}^{n_{g}} \mathcal{S}_{3 q j z}(y)\left(\zeta_{z}^{2}-\widehat{y}^{T} U_{z} \widehat{y}\right) \in \Sigma_{y}^{N \times N} \\
q: 1, \ldots, X ; j: 1, \ldots, n_{g} \quad(39) \\
{\left[\begin{array}{c}
P_{q}(\tilde{y})-\sum_{z=1}^{n_{g}} \mathcal{S}_{4 q j k z}(y)\left(\zeta_{z}^{2}-\widehat{y}^{T} U_{z} \widehat{y}\right) \quad(*) \\
M_{q j}^{[k]}(y)-L_{q j}^{[k]}(y) \\
q: 1, \ldots, X ; j: 1, \ldots, r ; k: 1, \ldots, m \quad(40) \\
\Sigma_{y}^{(N+1) \times(N+1)} \\
P_{q}\left(\tilde{y}_{0}\left(x_{q}\right)\right)-\widehat{y}\left(y_{0}\left(x_{q}\right)\right) \cdot \widehat{y}^{T}\left(y_{0}\left(x_{q}\right)\right) \succeq 0 \quad q: 1, \ldots, X \quad(41) \\
\Xi_{q i j}=T(y)\left(A_{q i}(y) P_{q}(\tilde{y})+B_{q i}(y) M_{q j}(y)\right)+(*) \\
-\sum_{k=1}^{n} \frac{\partial P_{q}(\tilde{y})}{\partial y{ }^{[k]}} A_{q i}(y)^{[k]} \widehat{y}
\end{array}\right.}
\end{gathered}
$$$$
\Upsilon_{q i j}=2 w^{T} \vartheta_{q i j}(y) B_{q i}^{T}(y) \sigma+w^{T} \vartheta_{q i j}(y) w-w^{T} L_{q j}(y) \sigma
$$

where $T(y)=\frac{\partial \widehat{y}(y)}{\partial y} \in \mathcal{R}_{y}^{N \times n}$ and $\Delta x=\frac{l_{2}-l_{1}}{X}$, then the closed-loop system (19) is locally exponentially stable with decay rate $\rho$ in the region (14), with controller (17) fulfilling bounds (18). The controller gains can be obtained as $K_{q j}(y)=M_{q j}(y) P_{q}^{-1}(\tilde{y})$.

Proof. Using the finite-differences method [39] for the spatial discretization, conditions in this theorem asymptotically approach ${ }^{3}$ those in Theorem 1 for large $X$, i.e., $K_{q j}(y)$ and $P_{q}^{-1}(\tilde{y})$ are good approximations to $K_{j}(y, x)$ and $P^{-1}(\tilde{y}, x)$ on the interval $\left[x_{q}-\Delta x / 2, x_{q}+\Delta x / 2\right]$. In this way, conditions (36) ensure (24) and (41) ensure (27).

Conditions (37)-38 are a particular convex implementation of 25], spatially discretized using a backward difference for $\partial P / \partial x$ [14]. On the one hand, multipliers $\mathcal{S}_{2}$ ensure that the spatial discretization of (33) holds in each $\Omega_{q}$. On the other hand, multipliers $\vartheta$ together with conditions (40) make (33) hold also in $\Pi$, as follows. We need to ensure the bound (18) inside each level set $\left\{\left(y, x_{q}\right): \widehat{y}^{T} P_{q}^{-1}(\tilde{y}) \widehat{y} \leq 1\right\}$. This, by the convex-sum property of $\mu_{j}(z)$ and Lemma 1 , is for $j: 1, \ldots, r$ :

$\eta_{k}^{2}-(*)^{T}\left(K_{q j}^{[k]}(y) \widehat{y}-\Psi^{[k]}\left(\nu_{q}\right)\right)-s_{q k}\left(1-\widehat{y}^{T} P_{q}^{-1}(\tilde{y}) \widehat{y}\right) \geq 0$

Now, assume that $\Psi(\nu)=\sum_{j} \mu_{j}(z) G_{j}(y) \widehat{y}$ when $\Psi(\nu) \neq$ 0 , being $G(y) \in \mathcal{R}_{y}^{m \times N}$ slack decision variables. Then,

\footnotetext{
${ }^{3}$ It is well established [38] that as $X$ increases, the closed-loop system that results from the PDE model plus an approximate finite-dimensional controller converges to 19 .
}

choosing $s_{q k}=\eta_{k}^{2}$ (classical S-procedure for quadratic LMIs) and doing the changes of variables $\widehat{y}=P_{q}(\tilde{y}) \sigma$, $L_{q j}(y)=G_{q j}(y) P_{q}(\tilde{y})$, the SOS conditions before lead to $\sigma^{T}\left(P_{q}(\tilde{y})-\eta_{k}^{-2}(*)^{T} \cdot\left(M_{q j}^{[k]}(y)-L_{q j}^{[k]}(y)\right)\right) \sigma$, which becomes 40 by Schur complement plus multipliers $\mathcal{S}_{4}$ to add local information of $\Omega_{q}$. So, what remains is checking the Lyapunov condition (21) together with ensuring $\Psi(\nu)=\sum_{j} \mu_{j}(z) G_{j}(y) \widehat{y}$ when $\Psi(\nu) \neq 0$ in . By Lemma 11 this is ensured adding the term $\Psi\left(\nu_{q}\right)^{T} \vartheta_{q}^{-1}(y)\left(\Psi\left(\nu_{q}\right)-\sum_{j} \mu_{j}(z) G_{q j}(y) \widehat{y}\right)$ to the spatiallydiscretized version of (33). By the convex-sum property and the changes of variables $\Psi(\nu)=w, \widehat{y}=P_{q}(\tilde{y}) \sigma$, the above can be expressed as

$$
\begin{aligned}
& {\left[\begin{array}{ll}
\sigma & w
\end{array}\right]\left[\begin{array}{cc}
\Lambda_{q i j}(y) & (*) \\
B_{q i}(y)+\vartheta_{q i j}^{-1}(y) L_{q j}(y) / 2 & \vartheta_{q i j}^{-1}(y)
\end{array}\right]\left[\begin{array}{c}
\sigma \\
w
\end{array}\right]} \\
& \in \Sigma_{y, \sigma, w}
\end{aligned}
$$

with $\Lambda_{q i j}(y)$ being the left-hand side of (37) (or 38) where corresponds) without the $\Upsilon$ terms. Hence, (42) becomes (37)-

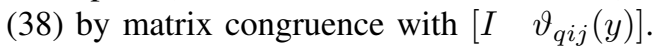

Finally, enforcing the Lyapunov level sets $\left\{\left(y, x_{q}\right)\right.$ : $\left.\widehat{y}^{T} P_{q}^{-1}(\tilde{y}) \widehat{y} \leq 1\right\} \subset \Omega_{q}$ is required to keep validity of the fuzzy-polynomial model. This, by similar argumentations to the ones discussed to obtain (40) and Positivstellensatz, leads to 39 , so 26) holds and the proof is complete.

Remark 7. Constraints (37)-(39) extend the generalized sector condition for control-input nonlinearity proposed in [40]. This extension allows all involved matrices being polynomial and parameter-dependent, which reduces the conservatism.

Corollary 1. Let $\delta \in \mathbb{R}^{+, m}$ be a set of parameters fixed a priori. If conditions

$$
\begin{gathered}
{\left[\begin{array}{cc}
P_{q}(\tilde{y})-\sum_{z=1}^{n_{g}} \mathcal{S}_{5 q j k z}(y)\left(\zeta_{z}^{2}-\widehat{y}^{T} U_{z} \widehat{y}\right) & (*) \\
M_{q j}^{[k]}(y) & \delta_{k}^{2} / \rho^{2}
\end{array}\right] \in} \\
\Sigma_{y}^{(N+1) \times(N+1)} q: 1, \ldots, X ; j: 1, \ldots, r ; k: 1, \ldots, m
\end{gathered}
$$

are added to the ones in Theorem 2 then the control-input variation with time is bounded by $\|\dot{\nu}\| \leq\|\delta\| \kappa+\gamma,(\gamma, \kappa)>0$.

Proof. By Theorem 2. $\|y\| \leq \kappa_{1} e^{-\rho t}\left\|y_{0}\right\|, \kappa_{1}>0$. By differentiating both sides with respect to time, one can obtain the bound $\rho\|y\| \leq\|\dot{y}\| \leq \rho \kappa_{2}\|y\|, \kappa_{2}>1$. Then, each controlinput derivative $\dot{\nu}_{q}=\sum_{j}\left(K_{q j}(y) T(y) \dot{y}+\sum_{k} \frac{\partial K_{q j}(y)}{\partial y^{[k]}} \dot{y}^{[k]} \widehat{y}\right)$ can be bounded by

$$
\begin{array}{r}
\left\|\dot{\nu}_{q j}\right\| \leq\left\|K_{q j}(y) T(y) \dot{y}\right\|+\gamma \leq\left\|K_{q j}(y) T(y)\right\| \cdot\|\dot{y}\|+\gamma \leq \\
\rho \kappa_{2}\left\|K_{q j}(y) T(y)\right\| \cdot\|y\|+\gamma \leq \rho \kappa_{2} \kappa_{3}\left\|K_{q j}(y) \widehat{y}\right\|+\gamma \quad(44)
\end{array}
$$

with $\gamma>0, \kappa_{3} \geq 1$ and the fact that $\partial K_{q j}(y) / \partial y^{[k]}$ are bounded in $\Omega_{q}$. Hence, ensuring $\delta^{T} \delta-\rho^{2}(*)^{T} \cdot\left(K_{q j}(y) \widehat{y}\right) \geq 0$ when $\widehat{y}^{T} P_{q}^{-1}(\tilde{y}) \widehat{y} \leq 1$ allows bounding $\|\dot{\nu}\| \leq\|\delta\| \kappa+\gamma$, $\kappa=\kappa_{2} \kappa_{3}$. This set inclusion is checked componentwise by (43) after the usual change of variables, Positivstellensatz and Schur complement, analogous to the development followed to obtain 40). 
Remark 8. Note that $\kappa_{2}$ is upper bounded by the highest decay rate achievable by the system at any time and trajectory, $\kappa_{3}$ is bounded thanks to the Cauchy-Schwarz inequality ${ }^{4}$ and $\gamma$ is proportional to the values of gains $K_{q j}$ and $y$, bounded too. Therefore, reducing $\delta$ in (43) lowers the gains $K_{q j}$, which themselves limits $\kappa$ and $\gamma$, thus reducing $\|\dot{\nu}\|$. In this way, $\delta$ can be treated as a design parameter to tune the aggressiveness of the controller.

Despite conditions (43) do not state a rigorous bound like the ones to ensure input saturation in Theorem 2, Corollary 1 addresses qualitatively an important limitation in practice: the actuator's speed (provided by its own dynamics or external safety limitations), often forgotten in most of theoretical LMI/SOS designs.

It is noteworthy to say that Assumption 1 is necessary to search for full $P(y, x)$ matrices, hence for more general Lyapunov functionals. However, removing the requirement of $\Theta=\alpha I$ is possible by restricting $P(y, x)$ in Theorem $1\left(P_{q}(\tilde{y})\right.$ in Theorem 2) to be diagonal. This conservative modification allows the treatment of systems with $\Theta=$ $\operatorname{diag}\left\{\alpha_{1}, \alpha_{2}, \ldots, \alpha_{n}\right\} \leq 0$. Observe also that if $\Theta \succ 0$, the results derived in this paper are also applicable if the boundary condition (10) is replaced by $y\left(l_{2}, t\right)=0$.

Note also that, differently from other SDLMI approaches such as [14] in the TS quadratic case, here there is no need of fixing a priori an initial $P_{0}(\tilde{y})$. Indeed, $P_{0}$ can be also decision variable, keeping convexity of the whole SOS problem. Fixing arbitrarily $P_{0}$ at the beginning may be conservative, decreasing thus the overall performance, as it influences the shape of the subsequent $P_{q}(\tilde{y})$ to be obtained.

\section{Optimization setups.}

Conditions in Theorem 2 and Corollary 1 can be used to pose some optimization problems such as:

a) Maximizing the guaranteed decay rate: If $\rho$ becomes decision variable to be maximized, Theorem 2 is not a convex SOS problem because constraints (37)-38 are bilinear in $\rho$ and $P_{q}(\tilde{y})$. However, this is a quasiconvex problem so-called generalized SOS problem [41]. In consequence, the global optimum can be computed via semidefinite programming by bisection on $\rho$, solving a feasibility problem at each step of the bisection algorithm.

b) Minimizing the controller aggressiveness: Given a minimum guaranteed decay rate, the smoothest controller fulfilling it from given initial conditions can be found by renaming $\delta_{k}^{2}=\lambda_{k}, \lambda$ new decision variables, and minimizing $J=\sum_{k=1}^{m} \lambda_{k} / \tau_{k}^{2}$, where $\tau_{k}$ is a normalizing factor which can be taken as, for instance, the corresponding span for the actuator $k$.

c) Maximizing the LDA estimate: Constraints (41) can be replaced by another set inclusion $\Theta_{i} \subset\left\{y: \widehat{y}^{T} P_{q}^{-1}(\tilde{y}) \widehat{y} \leq 1\right\}$, being $\Theta_{i}$ a region of initial conditions around the equilibrium. In particular, if $\Theta_{i}$ is a parameterized prefixed-shape set $H_{\lambda}=$ $\left\{y: \max _{i=1, \ldots, M} p_{i}(y) \leq \lambda\right\}$ an optimization problem can be set up maximizing the size parameter $\lambda$ to obtain the controller

\footnotetext{
${ }^{4}$ Useful here when the rows $K_{q j} T$ and $y$ are non-orthogonal, which is the case for a system to be controlled.
}

which maximizes the proven domain of attraction fulfilling the saturation constraints.

\section{Application Example}

The proposed distributed control design has been tested in simulation with the nonisothermal PFR in [10]. A PFR is an ideal flow reactor in which no back mixing occurs while a chemical reaction of the form $A \rightarrow \tilde{b} B$ takes place, being $\tilde{b}>0$ the stoichiometric coefficient. Thus, the composition of the reaction mixture changes along the length $x$ of the reactor, as represented in Figure 1

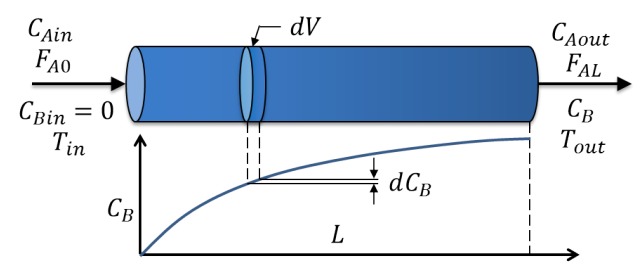

Fig. 1: Schema of a nonisothermal plug-flow reactor.

In Figure 1, $C_{A}$ and $C_{B}$ are the reactant and product concentrations respectively, $T$ denotes the reactor temperature, $T_{\text {in/out }}$ and $C_{A, \text { in } / \text { out }}$ are defined as the temperature and concentration of the inlet/outlet streams respectively, $F_{B}$ is the partial flow of product $\mathrm{B}$, and $L$ denotes the total length of the reactor. Under assumptions of perfect radial mixing, constant density and heat capacity of the reacting liquid, and negligible diffusive phenomena, a dynamic model of the process can be derived from material and energy balances in the form:

$$
\begin{aligned}
& \frac{\partial T}{\partial t}=-v \frac{\partial T}{\partial l}-\frac{k_{0} \Delta H}{\rho_{p} C_{p}} C_{A} \cdot e^{-\frac{E}{R T}}+\frac{4 h}{\rho_{p} C_{p} d}\left(T_{J}-T\right) \\
& \frac{\partial C_{A}}{\partial t}=-v \frac{\partial C_{A}}{\partial l}-k_{0} C_{A} \cdot e^{-\frac{E}{R T}} \\
& \frac{\partial C_{B}}{\partial t}=-v \frac{\partial C_{B}}{\partial l}+\tilde{b} \cdot k_{0} C_{A} \cdot e^{-\frac{E}{R T}}
\end{aligned}
$$

where $E, R, k_{0}, \Delta H, h$ and $d$ are the activation energy, the ideal-gas constant, the pre-exponential factor, the enthalpy of the reaction, the wall heat-transfer coefficient and the reactor diameter, respectively. $t, l$ denote the independent time and space variables. The control input is chosen to be the spatially uniform jacket temperature $T_{J}$. For simplicity the considered reaction is endothermic and a jacket is used to heat the reactor, so that the system is dissipative and open-loop stable. The process is subject to the boundary conditions: $T(0, t)=T_{i n}$, $C_{A}(0, t)=C_{A, i n}, C_{B}(0, t)=0$.

\section{A. Nonlinear dimensionless PDE model}

From 45-47), $C_{B}$ is known if $C_{A}$ and $T$ are known, so only the two first states will be considered henceforth. Hence, the following dimensionless states and input are introduced:

$$
\chi_{1}:=\frac{T-T_{i n}}{T_{i n}}, \chi_{2}:=\frac{C_{A, i n}-C_{A}}{C_{A, i n}}, \phi_{J}:=\frac{T_{J}-T_{i n}}{T_{i n}}
$$

Define also $x:=l / L$. Then an equivalent representation of (45)-(46) in variables (48) can be obtained, omitted for 
brevity. The reader is referred to [10] for a detailed description. Note that the dimensionless equilibrium profile $(t \rightarrow \infty)$ in one variable can be computed given a prefixed one for other variable. In this way, setting $T(x)=T_{i n}$ along the reactor in equilibrium for simplicity, the remaining profiles read:

$$
\chi_{1 e}(x)=0, \chi_{2 e}(x)=1-e^{-\frac{\beta_{2} L}{v} x}, \phi_{J e}(x)=-\frac{\beta_{1}}{b} e^{-\frac{\beta_{2} L}{v} x}
$$

with $\beta_{1} \triangleq \delta \beta_{2}, \beta_{2} \triangleq k_{0} \cdot e^{-\mu}$, and

$$
\mu \triangleq \frac{E}{R T_{i n}}, \delta \triangleq \frac{(-\Delta H) C_{A, i n}}{\rho_{p} C_{p} T_{i n}}, b \triangleq \frac{4 h}{\rho_{p} C_{p} d} .
$$

Numerical values for the above model parameters, used later in simulation, are in Table [

TABLE I: Model parameters for simulation.

\begin{tabular}{|l|l|}
\hline Parameters & Numerical values \\
\hline$v$ & $0.025 \mathrm{~m} / \mathrm{s}$ \\
\hline$L$ & $1 \mathrm{~m}$ \\
\hline$E$ & $11250 \mathrm{cal} / \mathrm{mol}$ \\
\hline$k_{0}$ & $10^{6} \mathrm{~s}^{-1}$ \\
\hline $4 h / \rho_{p} C_{p} d$ & $0.2 \mathrm{~s}^{-1}$ \\
\hline$C_{A, \text { in }}$ & $0.02 \mathrm{~mol} / \mathrm{L}$ \\
\hline$R$ & $1.986 \mathrm{cal} /(\mathrm{mol} . \mathrm{K})$ \\
\hline$T_{i n}$ & $340 \mathrm{~K}$ \\
\hline$\delta$ & 0.25 \\
\hline
\end{tabular}

Now, let us consider a new input vector $u(x, t)=\phi_{J}(x, t)-$ $\phi_{J e}(x)$ and the state transformation:

$$
y(x, t)=\left[\begin{array}{c}
\chi_{1}(x, t)-\chi_{1 e}(x) \\
\chi_{2}(x, t)-\chi_{2 e}(x)
\end{array}\right]
$$

Finally, the system (45)-(46) can be rewritten as (9) with:

$$
\begin{gathered}
\Theta=-\frac{v}{L} I, g(x)=[b, 0]^{T}, f(y, x)=\left[\beta_{1} f_{0}(y, x)-b y_{1},\right. \\
\left.\beta_{2} f_{0}(y, x)\right]^{T}, f_{0}(y, x)=\left(1-\chi_{2 e}(x)\right)\left[e^{\frac{\mu y_{1}}{1+y_{1}}}-1\right]-y_{2} e^{\frac{\mu y_{1}}{1+y_{1}}}
\end{gathered}
$$

\section{B. Fuzzy-polynomial PDE model}

The only non-polynomial term appearing in $f(y, x)$ is $\xi\left(y_{1}\right)=e^{\frac{\mu y_{1}}{1+y_{1}}}$. So, considering a modeling region

$$
\Omega=\left\{(y, x): y_{1}^{2} \leq \alpha^{2}, y_{2}^{2} \leq \iota^{2}, x \in[0,1]\right\}
$$

we can exactly represent $\xi\left(y_{1}\right)$ in $\Omega$ by

$$
\begin{aligned}
& \xi\left(y_{1}\right)=122.13 y_{1}^{2}+16.661 y_{1}+1+ \\
& \eta_{1}\left(y_{1}\right) \sup _{\Omega} T_{3}\left(y_{1}\right)+\eta_{2}\left(y_{1}\right) \inf _{\Omega} T_{3}\left(y_{1}\right)
\end{aligned}
$$

using the Taylor series [25] up to degree 3 . Here $\eta_{1}$ and $\eta_{2}$ are the nonlinear membership functions and $T_{3}$ is the Taylor's remainder of order 3 , computed as:

$$
\begin{gathered}
T_{3}\left(y_{1}\right)=\frac{\xi\left(y_{1}\right)-122.13 y_{1}^{2}-16.661 y_{1}-1}{y_{1}^{3}} \\
\eta_{1}\left(y_{1}\right)=\frac{T_{3}\left(y_{1}\right)-\inf _{\Omega} T_{3}\left(y_{1}\right)}{\sup _{\Omega} T_{3}\left(y_{1}\right)-\inf _{\Omega} T_{3}\left(y_{1}\right)}, \eta_{2}\left(y_{1}\right)=1-\eta_{1}\left(y_{1}\right)
\end{gathered}
$$

The choice of degree 3 for the vertex models achieves a good tradeoff between complexity and conservatism, i.e., the $3^{\text {th }}$-degree vertex models are significantly closer to $\xi$ than linear (TS) or quadratic ones. Figure 2 shows a comparison between the sector bounding of $\xi$ in $-0.2 \leq y_{1} \leq 0.2$ using $3^{t h}$-degree vertex models computed by Taylor series (Taylor O3) and linear ones (TS).

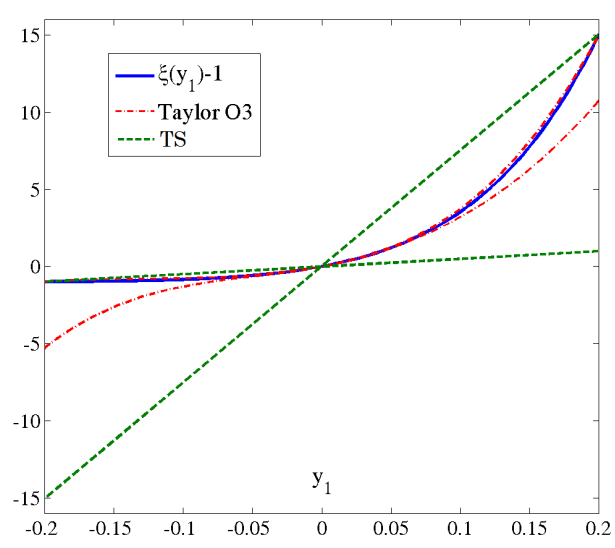

Fig. 2: Bounding $\xi$ with polynomial vertex models.

Assuming $y_{1}$ is measurable, this technique allows to rewrite the nonlinear PFR model in the fuzzy-polynomial form 16 .

\section{Simulation results}

We decided to compute a finite-dimensional controller (17) distributed in $X=30$ actuation points along the reactor's length, able to drive the PFR to the desired equilibrium profiles starting inside a region (50) defined by ${ }^{5} \alpha=0.1$ and $\iota=1$. This choice of modeling region covers the usual operation range of the plug-flow reactor near the chosen equilibrium.

The following choices have been made for numerical evaluation of Theorem 2 . $\widehat{y}=y$, fourth-degree Lyapunov matrices $P_{q}\left(y_{2}\right)$ and third-degree polynomial matrices $L_{q i}(y), M_{q i}(y)$. The user-defined scalars were set to $\rho=0.016$ (decay rate), $\varpi=0.1$ (saturation), $\delta=0.02$ (actuator rate) and $\epsilon=$ 0.0001 . The initial-condition profiles for simulation are chosen $y_{1}(x, 0)=0.07 \sin (2 \pi x L), y_{2}(x, 0)=0.5\left(e^{-\frac{\beta_{2} x L}{v}}-1\right)$. This means an initial reactor temperature fluctuating $\pm 25 \mathrm{~K}$ around the inlet temperature $T_{i n}$ and an initial concentration of reactant A starting at $C_{a, i n}$ in $x=0$ and decreasing exponentially up to $0.014 \mathrm{~mol} / L$ at $x=1$ (see Figures 3a 3b.

Then, the controller computed with the proposed method has been tested in simulation in closed loop with the nonlinear model of the plug-flow reactor. The evolution of the reactor states is depicted in Figure 3 Figure 4 shows the space-time evolution of the control input, the reactor jacket temperature. As it can be seen, the actuator saturates at its top and bottom limits during the first 2-3 seconds approximately, converging to the equilibrium profiles afterwards in a smooth way.

The discrete approximation of the LDA along the reactor's length $x$ is shown in Figure 5 . Each level set $y^{T} P_{q}^{-1}\left(y_{2}\right) y=1$, $q: 1, \ldots, X$, depicted on the right-hand side corresponds to a Lyapunov surface in $y\left(x_{q}\right)$ obtained using Theorem 2

\footnotetext{
${ }^{5}$ Note that this choice of $\Omega$ strictly should imply $U_{1}=\operatorname{diag}(1,0)$, $U_{2}=\operatorname{diag}(0,1)$ in 35 . However this may lead to numerical issues when computing the inverse, so a small tolerance $\epsilon \approx 0$ is used instead of zero in the main diagonal.
} 

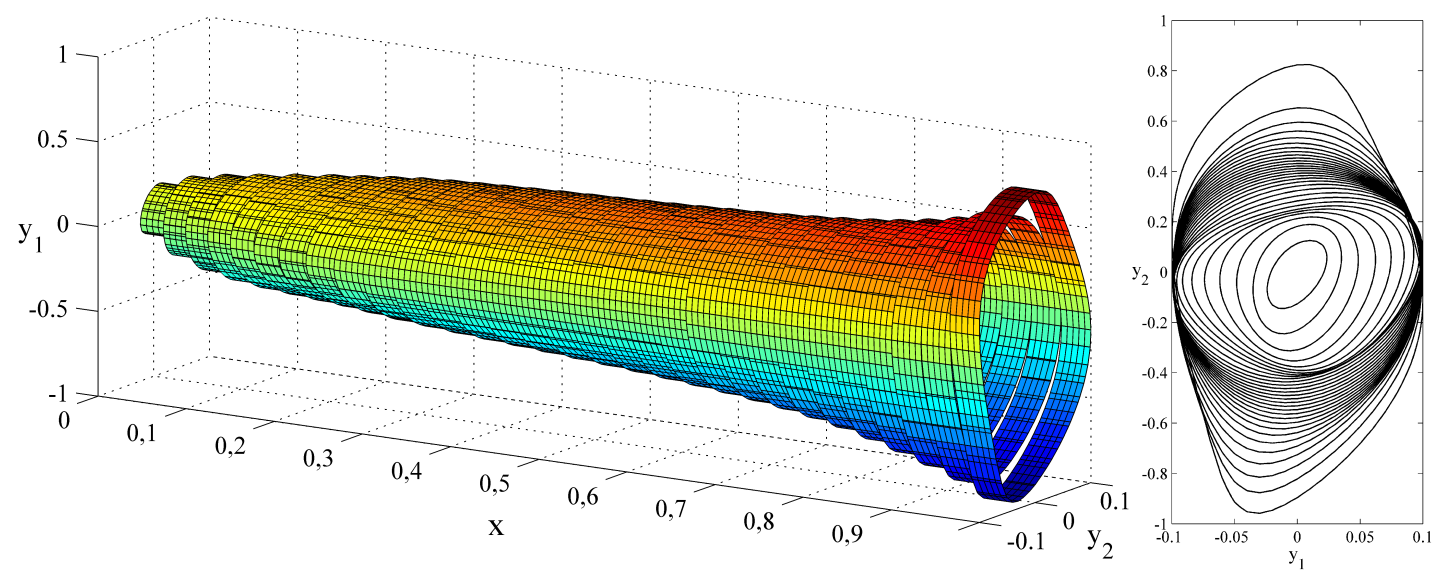

Fig. 5: Discrete approximation of the estimated LDA along the spatial variable (left) and its contour plots (right).

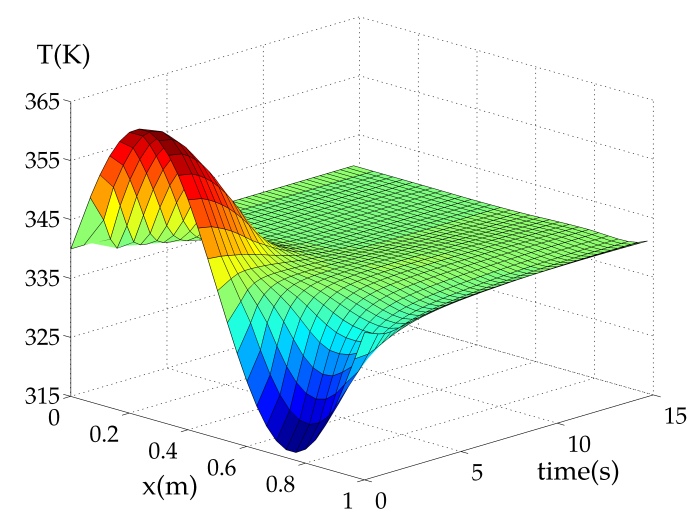

(a) Reactor temperature.

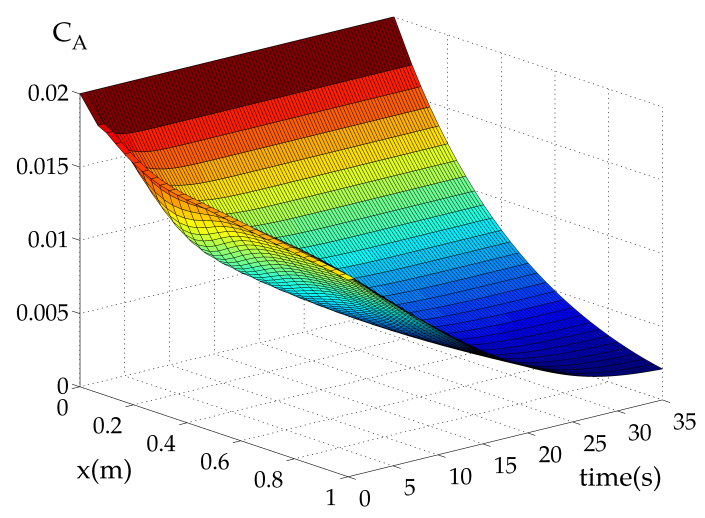

(b) Reactant concentration.

Fig. 3: Evolution of the reactor states in space and time.

\section{CONCLUSION}

In this paper we have proposed a systematic methodology for controller synthesis with input constraints for a class of distributed-parameter systems. The approach relies on fuzzypolynomial models to exactly represent non-polynomial nonlinearities in a local region, and available SOS-programming software to check for sufficient conditions to ensure exponential stability of the closed-loop system.

The approach has been used to compute a suitable nonlinear

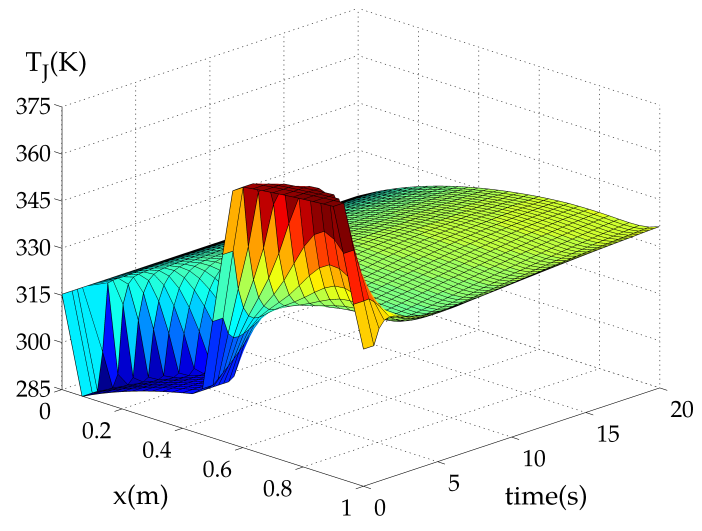

Fig. 4: Evolution of the controlled jacket temperature.

state feedback to control a nonisothermal PFR. Numerical simulations show that the obtained controller is able to drive the PFR from given initial conditions to the desired equilibrium profiles. The saturation limits are reached during the first seconds but, as expected, the controller gets a smooth response afterwards.

The computed control profile for the jacket temperature in simulation could be very useful in practice not only in terms of improving control but also in terms of process sizing: location of actuators, required control power, heat-transfer surfaces, etc.

Further research can be directed in several ways: addressing the problem of partially-unmeasurable states (output feedback), dealing with disturbances $\left(H_{\infty}\right.$, practical stability, etc.), reducing conservativeness (iterative SOS approaches for solving nonconvex problems) and extending the approach to other classes of PDE systems.

\section{ACKNOWLEDGMENT}

The research leading to these results has received funding from the European Union and from the Spanish Government (MINECO/FEDER DPI2015-70975-P). The first author especially thanks Prof. Antonio Sala for his endless support throughout his academic life. 


\section{REFERENCES}

[1] W. H. Ray, Advanced Process Control. New York: McGraw-Hill, 1981.

[2] A. D. Polyanin and V. F. Zaitsev, Handbook of nonlinear partial differential equations. CRC press, 2004.

[3] H. K. Rhee, R. Aris, and N. R. Amundson, First-order Partial Differential Equations: Vols. I and II. New Jersy: Prentice-Hall, 1986.

[4] H. N. Wu, J. W. Wang, and H. X. Li, "Design of distributed $H_{\infty}$ fuzzy controllers with constraint for nonlinear hyperbolic PDE systems," Automatica, vol. 48, pp. 2535-2543, 2012.

[5] P. D. Christofides, Nonlinear and robust control of PDE systems: Methods and applications to transport-reaction processes. Springer Science \& Business Media, 2012.

[6] D. Dochain, J. Babary, and N. Tali-Maamar, "Modelling and adaptive control of nonlinear distributed parameter bioreactors via orthogonal collocation," Automatica, vol. 28, no. 5, pp. 873 - 883, 1992.

[7] G. Bastin, J.-M. Coron, and S. O. Tamasoiu, "Stability of linear density-flow hyperbolic systems under PI boundary control," Automatica, vol. 53, pp. $37-42,2015$.

[8] R. Aguilar, A. Poznyak, R. Martínez-Guerra, and R. Maya-Yescas, "Temperature control in catalytic cracking reactors via a robust PID controller," Journal of Process Control, vol. 12, no. 6, pp. 695 - 705 , 2002.

[9] S. Mikhalevich, F. Rossi, F. Manenti, and S. Baydali, Robust PI/PID controller design for the reliable control of plug flow reactor, ser. Chemical Engineering Trans. AIDIC, 2015, vol. 43, pp. 1525-1530.

[10] I. Aksikas, J. J. Winkin, and D. Dochain, "Optimal LQ-feedback regulation of a nonisothermal plug flow reactor model by spectral factorization," IEEE Trans. on Automatic Control, vol. 52, pp. 11791193, 2007.

[11] I. Aksikas, A. Fusman, J. F. Forbes, and J. J. Winkin, "LQ control design of a class of hyperbolic PDE systems: Application to fixed-bed reactor," Automatica, vol. 45, pp. 1542-1548, 2009

[12] G. Valmorbida, M. Ahmadi, and A. Papachristodoulou, "Stability analysis for a class of partial differential equations via semidefinite programming," IEEE Trans. on Automatic Control, vol. 61, no. 6, pp. 1649-1654, June 2016.

[13] J. Wang, H. Wu, and H. Li, "Distributed proportional-spatial derivative control of nonlinear parabolic systems via fuzzy PDE modeling approach," IEEE Trans. on Systems, Man and Cybernetics, vol. 42, pp. $927-938,2012$.

[14] J. Wang, H. Wu, and H. Xiong, "Distributed fuzzy control design of nonlinear hyperbolic PDE systems with application to nonisothermal plug-flow reactor," IEEE Trans. on Fuzzy Systems, vol. 19, pp. 514 526,2011

[15] J.-W. Wang, H.-N. Wu, and H.-X. Li, "Guaranteed cost distributed fuzzy observer-based control for a class of nonlinear spatially distributed processes," AIChE Journal, vol. 59, no. 7, pp. 2366-2378, 2013.

[16] J.-W. Wang and H.-N. Wu, "Fuzzy output tracking control of semi-linear first-order hyperbolic PDE systems with matched perturbations," Fuzzy Sets and Systems, vol. 254, pp. 47 - 66, 2014.

[17] J. Qiu, S. X. Ding, H. Gao, and S. Yin, "Fuzzy-model-based reliable static output feedback $H_{\infty}$ control of nonlinear hyperbolic PDE systems," IEEE Trans. on Fuzzy Systems, vol. 24, no. 2, pp. 388-400, April 2016.

[18] M. S. Sadeghi, N. Vafamand, and M. S. Babaei, "Non-quadratic exponential stabilisation of non-linear hyperbolic partial differential equation systems," IET Science, Measurement Technology, vol. 8, no. 6, pp. $537-$ 545, 2014.

[19] P. Seiler, "SOSOPT: A Toolbox for Polynomial Optimization," ArXiv e-prints, Aug. 2013.

[20] A. Papachristodoulou and S. Prajna, "Analysis of non-polynomial systems using the sum of squares decomposition. in positive polynomials in control," Lecture Notes in Control and Information Sciences, vol. 312, pp. 23-43, 2005.

[21] G. Valmorbida, S. Tarbouriech, and G. Garcia, "Design of polynomial control laws for polynomial systems subject to actuator saturation," IEEE Trans. on Automatic Control, vol. 58, no. 7, pp. 1758-1770, 2013.

[22] J. L. Pitarch, A. Sala, J. Lauber, and T. M. Guerra, "Control synthesis for polynomial discrete-time systems under input constraints via delayedstate Lyapunov functions," Inter. Journal of Systems Science, vol. 47, no. 5, pp. 1176-1184, 2016.

[23] E. Meyer and M. M. Peet, "Stability analysis of parabolic linear PDEs with two spatial dimensions using Lyapunov method and SOS," in 2015 54th IEEE Conf. on Decision and Control, Dec 2015, pp. 1884-1890.
[24] K. Tanaka, H. Yoshida, H. Ohtake, and H. O. Wang, "A sum of squares approach to modeling and control of nonlinear dynamical systems with polynomial fuzzy systems," IEEE Trans. on Fuzzy Systems, vol. 17, pp. $911-922,2009$

[25] A. Sala and C. V. Ariño, "Polynomial fuzzy models for nonlinear control: a Taylor-series approach," IEEE Trans. on Fuzzy Systems, vol. 17, pp. 284-295, 2009.

[26] K. Tanaka, H. Yoshida, H. Ohtake, and H. O. Wang, "Guaranteed cost control of polynomial fuzzy systems via a sum of squares approach," IEEE Trans. on Systems, Man and Cybernetics B, vol. 39, pp. 561-567, 2009.

[27] J. L. Pitarch, A. Sala, and C. V. Ariño, "Closed-form estimates of the domain of attraction for nonlinear systems via fuzzy polynomial models," IEEE Tran. on Cybernetics, vol. 44, pp. 526 - 538, 2014.

[28] M. R. García, C. Vilas, L. O. Santos, and A. A. Alonso, "A robust multimodel predictive controller for distributed parameter systems," Journal of Process Control, vol. 22, no. 1, pp. 60 - 71, 2012.

[29] Z. Jarvis-Wloszek, R. Feeley, W. Tan, K. Sun, and A. Packard, "Control applications of sum of squares programming," in Positive Polynomials in Control, D. Henrion and A. Garulli, Eds. Springer, 2005, pp. 3-22.

[30] J. L. Pitarch, A. Sala, C. V. Ariño, and F. Bedate, "Domain of attraction estimation for nonlinear systems with fuzzy polynomial models," Revista Iberoamericana de Automática e Informática Industrial RIAI, vol. 9, no. 2, pp. $152-161,2012$.

[31] H. D. Tuan, P. Apkarian, T. Narikiyo, and Y. Yamamoto, "Parameterized linear matrix inequality techniques in fuzzy control system design," IEEE Trans. on Fuzzy Systems, vol. 9, p. 324 332, 2001.

[32] C. W. Scherer, "LMI relaxations in robust control," European Journal of Control, vol. 12, no. 1, pp. 3 - 29, 2006.

[33] C. M. Agulhari, R. C. L. F. Oliveira, and P. L. D. Peres, "LMI relaxations for reduced-order robust $H_{\infty}$ control of continuous-time uncertain linear systems," IEEE Trans. on Automatic Control, vol. 57, no. 6, pp. 1532 1537, June 2012.

[34] A. Sala and C. Ariño, "Asymptotically necessary and sufficient conditions for stability and performance in fuzzy control: Applications of Polya's theorem," Fuzzy Sets and Systems, vol. 158, pp. 2671-2686, jun 2007.

[35] C. W. Scherer and C. W. J. Hol, Matrix Sum-of-Squares Relaxations for Robust Semi-Definite Programs, ser. Mathematical Programming Ser. B-397. Springer Berlin / Heidelberg, jun 2006, vol. 107.

[36] A. Sala and J. L. Pitarch, "Optimisation of transient and ultimate inescapable sets with polynomial boundaries for nonlinear systems," Automatica, vol. 73, pp. 82-87, 2016.

[37] A. Pazy, Semigroups of linear operators and applications to partial differential equations, ser. Applied mathematical sciences. SpringerVerlag, 1983, vol. 44.

[38] M. J. Balas, "Finite-dimensional control of distributed parameter systems by Galerkin approximation of infinite dimensional controllers," Journal of Math. Analysis and Applications, vol. 114, no. 1, pp. 17 - 36, 1986.

[39] W. F. Ames, Numerical methods for partial differential equations, 3rd ed., ser. Computer science and scientific computing. Boston: Academic Press, 1992.

[40] J. M. G. da Silva and S. Tarbouriech, "Antiwindup design with guaranteed regions of stability: an LMI-based approach," IEEE Trans. on Automatic Control, vol. 50, no. 1, pp. 106-111, Jan 2005.

[41] P. Seiler and G. Balas, "Quasiconvex sum-of-squares programming," in in 49th IEEE Conf. on Decision and Control, 2010, pp. 3337-3342.

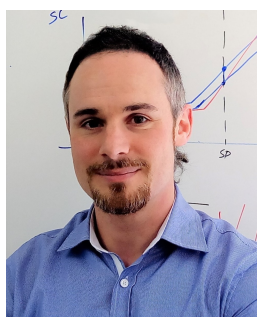

José Luis Pitarch received the M.Sc. degree in Industrial Engineering with honors from Jaume University of Castellón (Spain) in 2008. Then he was with BP Oil Refinery of Castellón as process control engineer in 2009. Afterwards he moved back to academia and he received the M.Sc. degree in Control and Industrial Informatics in 2010 and the Ph.D. degree in Control Engineering in 2013, both from Polytechnic University of Valencia (Spain). Currently he is with the University of Valladolid (Spain) as Junior Researcher. He is coauthor of 16 conference papers, 8 indexed (JCR) journal papers, a book and 3 book chapters. His research concerns real-time and robust optimization, data reconciliation, TS and fuzzy polynomial modelling and invariance-based control. 


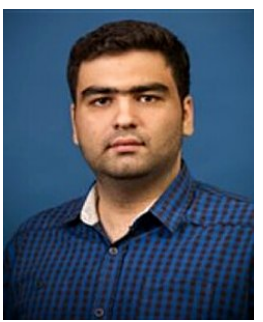

Mohsen Rakhshan received the B.Sc. in Electrical Engineering and the M.Sc. in Electrical Engineering both from Shiraz University of Technology (Iran). $\mathrm{He}$ is currently a Ph.D. student of Electrical Engineering at the University of Notre Dame (USA). His research interests are in control theory and mathematical modeling, biomechatronics, computational neuroscience and synthetic biology, among others.

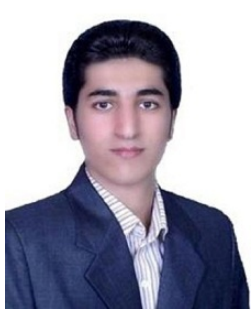

Mohammad Mehdi Mardani was born in Fars, Iran. He received his B.Sc. degree in Electrical and Electronics Engineering from the Fars Science and Research Azad University (Iran) in 2011 and the M.Sc. degree in Control Engineering from the Shiraz University of Technology (Iran) in 2014, where he currently works toward the Ph.D. degree in Control Engineering. His current research interests in the field of automatic control include fuzzy control of ODE/PDE systems, stability analysis, LMI/SOS programming, controller performance, sliding-mode control and consensus of multi-agent systems.

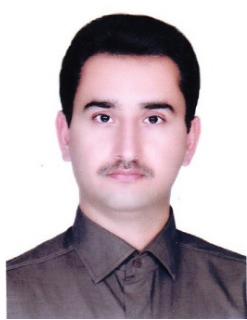

Mokhtar Shasadeghi received his B.Sc. degree in Electronics Engineering from Shiraz University in 1996 and the M.Sc. and Ph.D. degrees from Tarbiat Modares University, in 2001 and 2007, respectively, all in Iran. He is currently Associate Professor at the Shiraz University of Technology. His research interests include robust control, fuzzy control, time delay systems, linear matrix inequalities, sum-ofsquares decomposition and optimization. 